\title{
Correspondence
}

\section{Anaesthetic management of erythropoietic protoporphyria}

SIR-Asokumar et al. describe the uneventful management of a patient with erythropoietic protoporphyria for ventricular septal defect closure (1). The main emphasis of their treatment included precautions against the exposure of blood to light of wavelengths approximating $400 \mathrm{~nm}$ by using filters and the careful avoidance of drugs known to precipitate an acute porphyric crisis. They also discuss the intraoperative presentation and treatment of a porphyric crisis. I feel that I should point out that erythropoietic porphyrias are classified amongst the nonacute porphyrias, presenting with photosensitivity, peripheral paraesthesia and hepatic dysfunction but not the acute crisis the authors describe. Acute attacks only occur in four types of porphyria, namely acute intermittent porphyria, hereditary coproporphyria, variegate porphyria and plumboporphyria (2). Furthermore, erythropoietic porphyria is not associated with drug-precipitated crises, making the choice of anaesthetic agents less critical (2).

JOHN M. TORRANCE Pediatric Anesthesiology University of Michigan 1500 E. Medical Center Drive Ann Arbor, MI 48109-0211

USA

\section{References}

1 Asokumar B, Kierney C, James TW et al. Anaesthetic management of a patient with erythropoietic protoporphyria for ventricular septal defect closure. Ped Anaesth 1999; 9: 356-358.

2 Jensen NF, Fiddler DS, Striepe V. Anesthetic considerations in porphyrias. Anesth Analg 1995; 80: 591-599.

\section{Novel use of neonatal cuffed tracheal tube to occlude tracheo-oesophageal fistula}

SIR-we read the above case report by Greemberg et al. (1) with astonishment because we are convinced that the technique of attempting to occlude the fistula in a neonate with a tracheo-oesophageal fistula (TOF) using a cuffed tube, without knowing the exact location of the fistula, exposes the patient to the great risk of overinflating the stomach and intestinal tract.
The location of the fistula in patients with oesophageal atresia and TOF varies considerably (2). In more than $30 \%$ of these cases, the fistula is located in the right main stem bronchus, at the carina or close to the carina (3). This anatomical configuration puts all patients with a distal fistula in great danger if the technique described is used, namely, withdrawing a cuffed tracheal tube from the right main stem bronchus into the trachea, guided by auscultation only, and then inflating the cuff to try to occlude the fistula (Figure 1). In particular, if a carinal fistula is present, the inflated cuff will dilate the posterior wall of the trachea permitting an almost free flow of gas into the fistula and stomach. Furthermore, several pulmonary inflations using the reservoir bag may over inflate the gastro-intestinal tract to the point of duodenal rupture (two personal observations), thus necessitating a gastrostomy with its many disadvantages (4).

Only if a tracheoscopy is performed before intubation, demonstrating a mid tracheal fistula, can the described technique be considered safe. In this situation occlusion of the fistula is possible not only with a cuffed tracheal tube but also with an uncuffed one. The cuffed tube has the disadvantage of frequently being overinflated, damaging the structure of the trachea and does not permit the detection of a proximal fistula. If a cuffed tracheal tube with the cuff deflated is left in place for days, as described in this case report, the folds of the evacuated cuff may cause circular necrosis of the tracheal mucosa due to movement of the tube (5). From a surgical point of view a cuff could easily be sutured to large fistulae when ligation proves to be inappropriate.

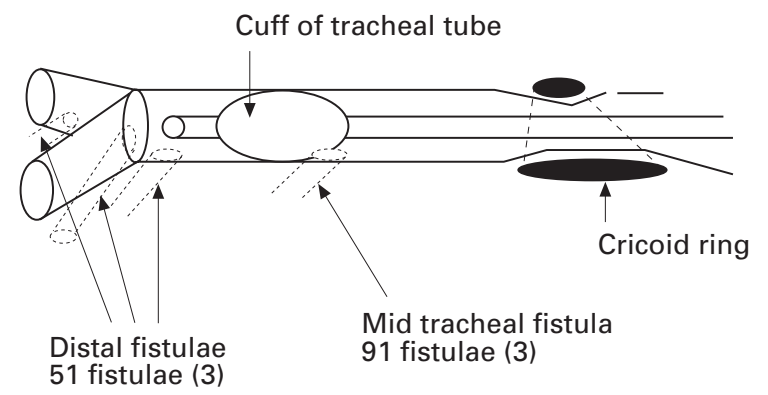

Figure 1

Drawing of a trachea in oesophageal atresia illustrating possible locations of distal fistulae (3). A cuffed tracheal tube will inevitably unfold fistulae and easily permit inflation of the stomach. 